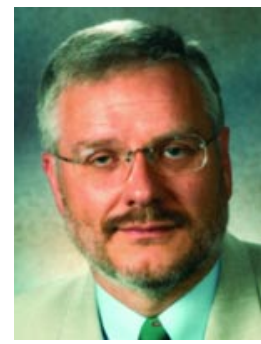

Ingo W. Husstedt

\title{
Opportunistische ZNS-Erkrankungen bei HIV und AIDS
}

\author{
Ingo W. Husstedt ${ }^{1}$, N. Gregor ${ }^{1}$, C. Kraemer ${ }^{1}$, A. Frese ${ }^{1}$, S. Kloska ${ }^{2}$, S. Evers ${ }^{1}$ \\ ${ }^{1}$ Klinik und Poliklinik für Neurologie, Division HIV-Infektion \\ 2 Institut für Klinische Radiologie, Universitätsklinikum Münster
}

psychoneuro 2004; 30 (12): 655-660

$\mathrm{B}$ ei Patienten mit HIV-Infektion werden grundsätzlich primäre und sekundäre Neuromanifestationen unterschieden $(6,7,10)$. Unter primären Neuromanifestationen (z.B. HIV-assoziierte Enzephalopathie und Myelopathie, HIVassoziierte Polyneuropathien) werden alle Erkrankungen des Nervensystems verstanden, die das Resultat der direkten HIV-Infektion des Nervensystems selbst darstellen. $\mathrm{Zu}$ den sekundären Neuromanifestationen zählen alle Erkrankungen, die sich infolge des HIV-induzierten, progredienten Immunmangelsyndroms entwickeln (opportunistische Infektionen, wie z.B. die zerebrale Toxoplasmose, Lymphome).

Neuropathologische Untersuchungen weisen Neuromanifestationen bei bis zu $90 \%$ aller HIV-Infizierten nach und es besteht eine direkte Korrelation zwischen der Progredienz der HIV-Infektion und Neuromanifestationen $(2,10,11)$.

Als Konsequenz der hocheffektiven Medikamentenkombinationen hat sich die Überlebenszeit im AIDSStadium auf mehrere Jahre verlängert und die klassischen Formen opportunistischer Infektionen werden in Deutschland meistens noch bei unbehandelten HIV-Infizierten mit ausgeprägtem Immundefekt oder aber bei Immigranten beobachtete.

Nach Einführung der hochaktiven antiretroviralen Therapie (HAART) als Standard-Therapie der HIV-Infektion wurde ein drastischer Rückgang von opportunistischen Infektionen beobachtet. Die Inzidenz von neu aufgetretenen Erkrankungen des zentralen Nervensystems reduzierte sich von 5,9 pro 100 Personen im Jahr 1994 auf 0,5 im Jahr 2002. Die schweren Verläufe opportunistischer zerebraler Infektionen treten in den hochentwickelten Industrieländern nun bei Patienten mit schwerem Immundefekt oder aber unbekannter HIV-Infektion auf. Unter den opportunistischen Infektionen mit fokalen Läsionen sind die Toxoplasmose und die progressive multifokale Leukoenzephalopathie führend. Unter den diffusen Erkrankungen ist die zerebrale Kryptokokkose häufig, aber im Prinzip können alle Infektionen, mit denen Kontakt bestanden hat, aufgrund des schweren Immunmangelsyndroms als opportunistische Infektion erneut reaktiviert werden. Die Differentialdiagnostik der opportunistischen Infektionen erfolgt durch neuroradiologische Untersuchungen wie Kernspintomographie und die Liquoranalyse unter Einsatz der Polymerasekettenreaktion. Nuklearmedizinische Verfahren sind hilfreich zur Abgrenzung gegenüber dem primär zerebralen Lymphom, der wichtigsten Differentialdiagnose der zerebralen Toxoplasmose. Bei opportunistischen Infektionen, die nicht ausreichend mit konventionellen konservativen Verfahren abzuklären sind, ist der Einsatz der stereotaktischen Biopsie sinnvoll und indiziert. Die Prognose opportunistischer Infektionen hat sich unter der Kombination von HAART und rechtzeitig beginnender konsequenter Therapie der opportunistischen Infektion wesentlich verbessert.

Für den Neurologen haben sich neue Krankheitsbilder wie z. B. neurologische Immunrekonstitutionserkrankungen entwickelt. Seit 1996 ist auch in Deutschland die hochaktive antiretrovirale Therapie mit der Kombination von mindestens drei Retrovirustatika unterschiedlicher Substanzklassen (hochaktive antiretrovirale Therapie, HAART) die Basistherapie.

Tabelle 1 stellt Inzidenzraten von Neuromanifestationen unter den verschiedenen antiretroviralen Therapieverfahren dar (15).
Opportunistische Infektionen entwickeln sich aufgrund der verschlechterten zellulären Immunabwehr und der abnehmenden spezifischen humoralen Immunantwort auf Antigene. Im Vergleich zu Immunkompetenten sind die Verläufe von Meningitiden und Enzephalitiden bei HIV-Infizierten nicht so charakteristisch. Hierdurch können differentialdiagnostische Schwierigkeiten entstehen, insbesondere, wenn zudem typische zerebrale strukturelle Läsionen mit neuroradiologischen Methoden nicht nachweisbar sind. Auf- 
Tab. 1 Inzidenzrate HIV-assoziierter neurologischer Manifestationen

\begin{tabular}{lccc} 
Erkrankungen & $\begin{array}{c}1990-1992 \\
\text { Monotherapie }\end{array}$ & $\begin{array}{c}1993-1995 \\
\text { Dualtherapie }\end{array}$ & $\begin{array}{c}\text { 1996-1998 } \\
\text { HAART }\end{array}$ \\
\hline HIV-assoziierte Enzephalopathie & 21,1 & 17,8 & $10,5^{*}$ \\
\hline Kryptokokken-Meningitis & 5,0 & 2,5 & $1,5^{*}$ \\
\hline Zerebrale Toxoplasmose & 5,4 & 3,8 & 2,2 \\
\hline PML & 2,0 & 1,8 & 1,5 \\
\hline Primär zerebrales Lymphom & 2,8 & 4,3 & $0,4^{*}$
\end{tabular}

Inzidenzrate HIV-assoziierter neurologischer Manifestationen unter verschiedenen retroviralen Therapieverfahren (Inzidenzraten pro 1000 Personen/Jahr; * $\mathrm{p}<0.05$ bei Regressionsanalyse) (15)

grund der schlechten Ausgangssituation bei opportunistischen zerebralen Infektionen ist es sinnvoll, rasch nach der klinischen Gesamtkonstellation $\mathrm{zu}$ behandeln und nicht Tage bis zum Eintreffen irgendwelcher Untersuchungsergebnisse verstreichen zu lassen.

Im Gegensatz zu den primären Neuromanifestationen setzen opportunistische Infektionen hochakut und dramatisch ein. Tabelle 2 listet typische Symptome und klinische Befunde bei opportunistischen Infektionen auf.

\section{Abb. 1 Bihemisphärische subkortikale Läsionen sowie Läsion im Bereich der rechten Stammganglien}
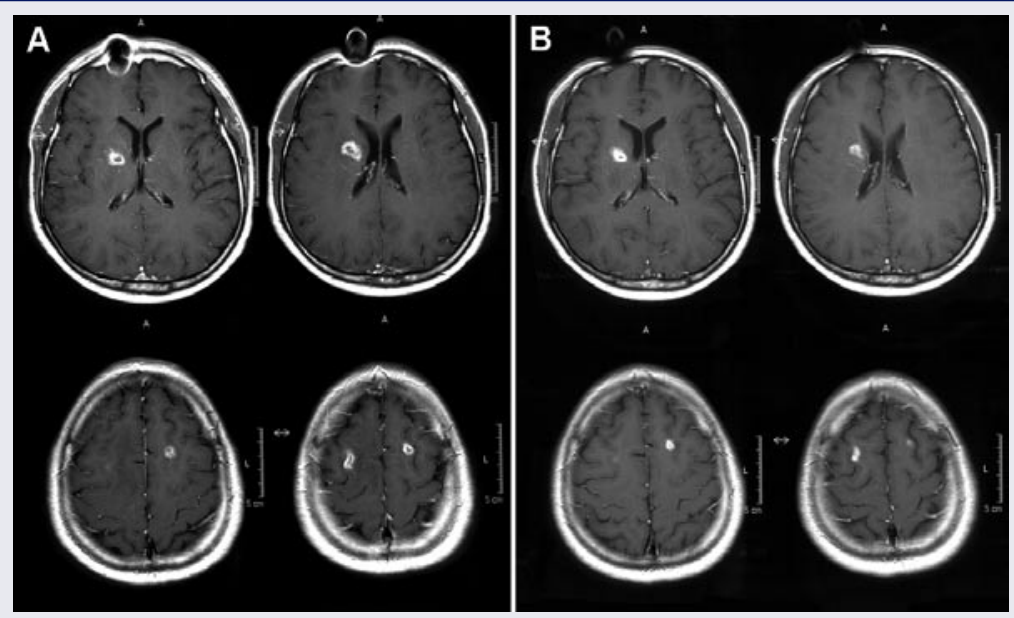

Die initiale MRT-Untersuchung (A) zeigt in der axialen T1-gewichteten Sequenz mehrere bihemisphärische subkortikale Läsionen sowie eine Läsion im Bereich der rechten Stammganglien. Sämtliche Läsionen zeigen eine kräftige, randständige Kontrastmittelanreicherung als typisches Zeichen einer zerebralen Toxoplasmose.

In der MRT-Verlaufskontrolle nach drei Monaten (B) sind unter Therapie sämtliche vorbestehenden Läsionen in der axialen T1-gewichteten Sequenz größenregredient. Es besteht jedoch weiterhin eine randständige Kontrastmittelanreicherung der Läsionen als Ausdruck einer fortbestehenden BlutHirn-Schrankenstörung. Neu aufgetretene Läsionen sind nicht nachweisbar. Nebenbefundlich Metallartefakt in der Schädelkalotte rechts frontal. ödem ist typisch, die Läsionen finden sich akzentuiert in den Stammganglien und subkortikal. Die Magnetresonanztomographie mit Gadolinium-DTPA ist wesentlich sensitiver und stellt oft Herde dar, die in der Computertomographie nicht entdeckt werden können. Vor jeder Lumbalpunktion ist eine neuroradiologische Bildgebung notwendig, um keine intrakranielle Druckerhöhung zu übersehen.

Die Liquoranalyse ergibt bei $50 \%$ der Patienten eine Pleozytose. Eine Gesamtproteinerhöhung wird bei 60 bis 70\% gefunden. Die Glukose im Liquor ist bei einigen Patienten herabgesetzt im Vergleich zu den Werten im Serum. Die Toxoplasmose-PCR im Liquor ist hilfreich, weist jedoch eine relativ schlechte Sensitivität auf. Zusätzlich zum Nachweis der Toxoplasmose dient die Liquoranalyse dem Ausschluss anderer opportunistischer Infektionen und Tumoren. Das Resultat der Liquoranalyse kann jedoch trotz nachgewiesener Toxoplasmose unauffällig sein. Serologisch lässt sich oft spezifisches IgG nachweisen, jedoch keine IgM-Antikörper.

Der sofortige Beginn einer Kombinationstherapie ist bereits bei begründetem Verdacht auf eine zerebrale Toxoplasmose indiziert. Dosierungsangaben sind in der Literatur nicht einheitlich.

Folgende Initialkombination ist allgemein akzeptiert:

- Pyrimethamin: initial am 1. Tag $200 \mathrm{mg}$, anschließend $100 \mathrm{mg} / \mathrm{Tag}$

- Sulfadiazin: 4 mal 1,0-1,5 g/Tag oral

- Folinsäure: 15 mg/Tag.

Abbildung 1 stellt den typischen Verlauf einer zerebralen Toxoplasmose unter Therapie dar. Tabelle 3 zeigt Therapiealternativen bei Allergien oder Resistenz und Erhaltungstherapien nach Remission. Die Therapie wird solange fortgeführt, bis keine Läsion mehr nachweisbar ist.

Differentialdiagnostisch ist das primär zerebrale Lymphom vorrangig zu berücksichtigen, welches im allgemeinen jedoch bei noch niedrigeren $\mathrm{CD}^{+}{ }^{+}$-Zellzahlen auftritt $(2,4$, $9,10,13)$. 
Bei unkomplizierten Verläufen ist bereits mit einem Ansprechen auf die Therapie innerhalb von ein bis zwei Wochen zu rechnen, eine Ausheilung kann nach sechs bis acht Wochen erreicht werden, sich gegebenenfalls auch über ein bis zwei Jahre hinziehen.

Die stereotaktische Biopsie ist in Fällen mit uncharakteristischen neuroradiologischen Läsionen, atypischem klinischen Verlauf, rascher Progredienz und fehlender Remission unter der Primärtherapie nach zwei bis drei Wochen indiziert.

Untersuchungen zur Korrelation klinisch apparativer Differentialdiagnosen mit neuropathologischen Diagnosen bei Patienten mit opportunistischen Infektionen im AIDS-Stadium ergaben, dass eine hohe Übereinstimmung zwischen den klinischen Verdachtsdiagnosen und den neuropathologischen Diagnosen besteht. Die beste Sensitivität erzielte die klinische Verdachtsdiagnose für die progressive multifokale Leukoenzephalopathie, während gerade für die zerebrale Toxoplasmose klinisch die schlechteste Sensitivität und Spezifität bestand. Die Ergebnisse zeigen, dass die progressive multifokale Leukoenzephalopathie mit sehr großer Sicherheit klinisch diagnostiziert werden kann, wogegen bei Verdacht auf zerebrale Toxoplasmose oder aber für die wichtigste Differentialdiagnose, das primär-zerebrale Lymphom, die Schwelle für eine Biopsie niedrig anzusetzen ist (1).

\section{Progressive multifokale Leukoenzephalopathie (PML)}

Etwa 80 bis $90 \%$ der Bevölkerung haben Antikörper gegen das Polyoma-Virus JC. Die mit diesem Erreger assoziierte progressive multifokale Leukenzephalopathie (PML) tritt bei zirka 4 bis $5 \%$ aller HIV-Infizierten auf $(4,8,13)$ und ist vier- bis fünfmal häufiger als bei Patienten nach Nierentransplantation.

Oft treten Gesichtsfelddefekte und Dysarthrien auf. Hirnorganische Psychosyndrome und epileptische Anfälle sind dagegen selten.

Die Computertomographie ist wenig sensitiv und ergibt hypodense, keine Kontrastmittel-aufnehmende Läsionen. Die neuroradiologische Diagnostik der progressiven multifokalen Leukenzephalopathie (PML) ist eine Domäne der Kernspintomographie. In $\mathrm{T}_{1}$-gewichteten Aufnahmen zeigen sich homogene hypointense Herde, die den Kortex auszusparen scheinen und von gleicher Ausdehnung in der $\mathrm{T}_{2}$-Wichtung sind. Die Läsionen erscheinen hypointens in der $\mathrm{T}_{1}$ - und hyperintens in der $\mathrm{T}_{2}$-Wichtung, nehmen im Allgemeinen kein Kontrastmittel auf und sparen den Kortex aus. Im Randbereich der Läsionen sind bei bis zu $10 \%$ geringe Konrastmittelaufnahmen möglich. Die Läsionen finden sich bevorzugt in parieto-okzipitialer Lokalisation, bei 30\% in der hinteren Schädelgrube.

Die Liquoranalyse ergibt in der Routineuntersuchung meistens Normalbefunde. Der Nachweis von JC-Virus-DNA durch die PCR im Li-
Tab. 2 Symptome und klinische Befunde bei opportunistischen Infektionen

- Krankheitsgefühl

- Fieber

- Kopfschmerzen

- Epileptische Anfälle

- Hirnorganisches Psychosyndrom

- Meningismus

- Fokale neurologische Zeichen wie

- Hemiparese

- Aphasie

- Hemianopsie

- Ataxie

- Gangstörungen

- Zerebrale Toxoplasmose

quor weist eine Sensitivität von bis zu $90 \%$ und eine Spezifität von mehr als $90 \%$ auf und ist damit sehr aussagekräftig. Alternativ kann der Nachweis einer intrathekalen Synthese von VP1-spezifischen IgG-Antikörpern durchgeführt werden (Sensitivität ca. 76\%, Spezifität 96\%) (13). Die typischen klinischen Befunde, die neuroradiologischen Ergebnisse sowie die Ergebnisse der PCR-Analyse führen dazu, dass in den meisten Fällen auf eine stereotaktische Biopsie verzichtet werden kann.

Die PML hatte vor der HAARTÄra eine mittlere postdiagnostische Überlebenszeit von drei bis sechs Monaten. Unter Immunrekonstitution kommt es z. T. zur Remission und zur Stabilisierung über mehrere Jahre. Die Kombination von HAART und Cidofovir scheint den Therapie-

\section{Tab. 3 Alternative Medikamente und Erhaltungstherapie der zerebralen Toxplasmose}

\section{Akuttherapie}

Alternative (bei Sulfonamidunverträglichkeit):

Clindamycin (4x 600 mg/d i.v. oder p.o.) + Pyrimethamin (Dosis wie oben)

\section{Weitere Alternativen:}

Clarithromycin p.o. (2x 0,5 bis $1 \mathrm{~g} / \mathrm{d}$ ) oder Azithromycin p.o. (1x 0,5 bis $1 \mathrm{~g} / \mathrm{d}$ ) zusammen mit Pyrimethamin und Folinsäure

Atovaquone p.o. (4x $750 \mathrm{mg} / \mathrm{d})$ als Monotherapie
Nebenwirkungen

Pyrimethamin:

Hautausschlag, makrozytäre Anämie, selten Kopfschmerzen, Anfälle.

Sulfadiazin, Cotrimoxazol:

Toxisch-allergische Hautreaktionen,

Kristallurie. Weniger bedeutsam:

Myalgien, Arthralgien

Clindamycin:

Gastroenterologische Komplikationen, pseudomembranöse Colitis
Erhaltungstherapie nach Remission

Pyrimethamin p.o. $(50 \mathrm{mg} / \mathrm{d})$ oder

Cotrimoxazol p.o. $(960 \mathrm{mg} / \mathrm{d}+15 \mathrm{mg}$

Folinsäure $2 x$ pro Woche)

Atovaquon $750-1500 \mathrm{mg}$

Absetzen der Sekundärprophylaxe bei stabiler T4-Zellzahl > 200/ $\mu$ l über 6 Monate möglich*

* Da noch keine ausreichende Datenlage vorhanden, kann dieses Vorgehen noch nicht allgemein empfohlen werden 
erfolg und die mittlere Überlebenszeit wesentlich zu verbessern $(3,5$, $8,10,13)$. Cidofovir wird initial zweimal jede Woche, danach alle 14 Tage appliziert, wobei die Dauer dieser Therapie nicht sicher geklärt ist.

Sinnvoll ist es, zu therapieren, bis unter der Kombination mit HAART und Cidofovir eine stabile Phase fehlender Progredienz über mindestens sechs bis acht Wochen erreicht worden ist. Unter kontinuierlicher Verlaufskontrolle, auch unter dem Einsatz der Kernspintomographie, wird dann abgewartet, ob eine weitere Regredienz oder aber Progredienz eintritt, sodass eine erneute Therapieaufnahme mit Cidofovir indiziert ist.

Mit der Kombination von HAART und Cidofovir scheint sich die Prognose wesentlich verbessert $\mathrm{zu}$ haben, obwohl der Stellenwert der Kombinationstherapie von HAART und Cidofovir bei PML noch nicht endgültig geklärt ist $(3,5,9,10,13)$.

\section{Kryptokokken-Meningo- enzephalitis}

Die Kryptokokken-Meningoenzephalitis ist die häufigste zerebrale Pilzinfektion bei HIV-infizierten Patienten, die Inzidenz beträgt $3 \%$. Sie tritt bevorzugt bei $\mathrm{CD}^{+}{ }^{+}$Zellen $<100 \mu$ auf.

Nach initial inapparentem Befall der Lunge tritt eine hämatogene Streuung in die Meningen ein (10, 13, 16). Die klinische Symptomatik ist durch eine Meningitis mit Fieber, Kopfschmerzen, Übelkeit und Erbrechen gekennzeichnet. Epileptische Anfälle sind selten, fokalneurologische Ausfälle finden sich wesentlich seltener als bei den anderen opportunistischen Infektionen und können Hinweise auf ein Kryptokokkom sein. Eine zunehmende Vigilanzminderung und progrediente Kopfschmerzen weisen meistens auf einen Hydrozephalus hin.

Neuroradiologische Methoden ergeben bei bis zu 90\% der Betroffenen Normalbefunde; gelegentlich sind Kryptokokkome nachweisbar. Im Liquor findet sich eine leichte bis mäßige, vorwiegend lymphozytäre Pleozytose, das Gesamtprotein ist erhöht und die Glukosekonzentration im Liquor erniedrigt. Die Li-
Abb. 2 Kryptokokke im Liquor zerebrospinalis bei einem
Patienten mit Kryptokokken-Meningoenzephalitis



Kryptokokke dargestellt in der Fuchs-Rosenthal-Zählkammer. Vergrößerung 25-fach, Färbung mit Methylviolett.

quorzytologie insbesondere im $\mathrm{Tu}-$ schepräparat ergibt bei bis zu $90 \%$ den Direktnachweis der typischen Kryptokokken, die sich in „sombreroartiger" Form darstellen und eventuell mit Lymphozyten verwechselbar sind. Abbildung 2 zeigt das typische Bild von Kryptokokken in der Fuchs-Rosenthal-Zählkammer.

Während der Lumbalpunktion ist immer eine Liquordruck-Messung notwendig. Als Pathomechanismus einer Liquordruckerhöhung wird eine Verlegung der Liquorresorptionswege durch Kryptokokken angenommen. Ein erhöhter Liquordruck kann bei positivem Kryptokokken-Antigen im Serum - auch ohne typische laborchemische Veränderungen im Liquor - ein Hinweis auf eine Kryptokokken-Meningoenzephalitis sein. Eine Senkung des Liquordruckes ist dringend indiziert. Hierzu werden in erster Linie wiederholte Lumbalpunktionen eingesetzt. Auch eine Ventrikeldrainage kann appliziert werden, falls wiederholte Lumbalpunktionen nicht toleriert werden.

Der Nachweis des Kryptokokken-Antigens im Serum und im Liquor hat eine Sensitivität von na- hezu 100\%. Die Bestimmung des Kryptokokken-Antigens im Serum kann daher als Screening-Methode eingesetzt werden.

Die Therapie der KryptokokkenMeningoenzephalitis erfolgt mit einer i.v.-Kombinationstherapie, bestehend aus Amphotericin B i.v. (0,7-1,0 mg/kg/Tag) plus Fluconazol i.v. oder oral ( 2 x 200 bis 400 mg/Tag), dazu Flucytosin (150 $\mathrm{mg} / \mathrm{kg} /$ Tag auf 4 Dosen verteilt) über zwei bis sechs Wochen oder bis zur sterilen Liquorkultur. Danach ist eine lebenslange Rezidivprophylaxe mit Fluconazol (200-400 mg oral/Tag) oder alternativ - bei Unverträglichkeit - mit Itraconazol (200 mg/Tag) notwendig.

\section{Seltenere opportunistische Infektionen}

Auch das Epstein-Barr-Virus kann eine Meningoenzephalitis verursachen. Die Häufigkeit wird mit 0,7-5,5\% angegeben. Die klinischen Symptome einer Epstein-Barr-VirusMeningoenzephalitis unterscheiden sich nicht von anderen viralen opportunistischen Infektionen. Seltene klinische Manifestationen stellen eine Cerebellitis mit Ataxie und eine Querschnittsmyelitis dar. 
Infektionen mit Listeria monocytogenes treten bei Patienten mit HIV-Infektion gelegentlich auf. Meistens sind Patienten im fortgeschrittenen Stadium der HIV-Infektion betroffen. Oft besteht eine Meningitis, auch Abszesse werden gefunden. Listeria monocytogenes lokalisiert sich in höherem Maße im Hirnstamm als andere bakterielle opportunistische Infektionen. Die Mortalität beträgt bis zu 30\%.

Die Histoplasmose, die Kokkzidioidomykose und die Blastomykose weisen in Endemiegebieten Afrikas und Asiens sowie in einigen tropischen bzw. subtropischen Regionen Amerikas eine hohe Inzidenz auf und sollten insbesondere bei Immigranten aus diesen Regionen differentialdiagnostisch berücksichtigt werden.

Eine weitere relevante Pilzinfektion ist die Aspergillose. Sie kommt vorwiegend bei Patienten mit extremer Abwehrschwäche vor (CD4-

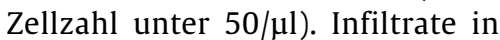
den Lungen sind häufig, aber auch eine Aussaat in das Zentralnervensystem wird beobachtet. Isolierte Hirnabszesse und eine basale Meningitis können auftreten. Der Ausgangsherd kann eine Sinusitis sein. Auch Nokardia asteroides kann durch hämatogene Aussaat bei CD4+-Zellen $<200 \mu$ in seltenen Fällen als opportunistische Infektion auftreten. Computer- und kernspintomographisch zeigen sich bei beiden Erkrankungen multiple girlandenförmig Kontrastmittel aufnehmende Läsionen mit perifokalem Ödem. Bei Abzessen durch Aspergillin lässt sich z.T. neuroradiologisch eine Dreischichtung nachweisen. Die Aspergillose wird mit Amphoterizin B iv. und Flucytosin behandelt, Alternativen stellen Itraconazol oder liposomales Amphotericin B dar. Für die Behandlung der Nokardiose hat sich Cotrimoxazol oder Meronem bewährt. Während die Infektion mit Aspergillus fast immer letal verläuft, kann bei der Nokardiose bei rechtzeitiger und früher Therapie eine Defektheilung eintreten. Die Erhaltungstherapie ist nicht sicher geklärt, bei Aspergillose kann Itraconazol und bei der Nokardiose Folinsäure eingesetzt werden (13).

\section{Differenzialdiagnose primär zerebrales Lymphom des ZNS}

Es ist davon auszugehen, dass bei ein bis zwei Prozent aller HIV-Infizierten ein primäres, zerebrales Non-Hodgkin-Lymphom (NHL) auftritt, unter HAART ist die Inzidenz vermutlich rückläufig $(2,9,10,11)$. Im Gegensatz zu den systemischen Lymphomen treten die primär zerebralen in weiter fortgeschrittenen Stadien der HIV-Infektion auf, meistens bei $\mathrm{CD}^{+}{ }^{+}$-Zellzahlen $<50 / \mu \mathrm{l}$.

Prinzipielle Unterschiede bezüglich der Symptome und klinischen Untersuchungsbefunde bestehen im Vergleich zu opportunistischen Infektionen mit fokalen Läsionen nicht, oft sind Patienten mit NHL jedoch in deutlich schlechterem klinischen Allgemeinzustand.

Pathognomonische neuroradiologische Befunde existieren für primär zerebrale Lymphome nicht. Oft zeigen sich ringförmige kontrastmittelanreichernde Läsionen im Corpus callosum, die raumfordernden Charakter haben und zur Verdrängung der Ventrikel sowie zur Verlagerung der Mittellinie führen. Ein Ödem ist oft nur gering ausgeprägt, ringförmige Strukturen bestehen in $10 \%$ der Fälle. Viele primäre zerebrale Lymphome haben Kontakt zum Ependym oder zu den Meningen, bei 50 bis 60\% der Betroffenen liegen multiple Läsionen vor. Die Kernspintomographie mit Gadolinium ist wesentlich sensitiver als die Computertomographie. Zur weiteren Differentialdiagnostik kann das Thallium 201-SPECT und die Positronenemissionstomographie eingesetzt werden, in der sich eine Anreicherung der Tracer findet. Die Spezifität dieser Untersuchung ist jedoch relativ gering $(13,14)$.

Die Liquoranalyse ist oft unergiebig und nur bei einem Drittel der Betroffenen zeigen sich typische Veränderungen. Es ergeben sich eine lymphozytäre Pleozytose mit Lymphoblasten und eine Erhöhung des Gesamtproteins, gelegentlich kann die Glukose im Liquor erniedrigt sein. In der Liquorzytologie finden sich homogene Lymphoblasten mit intranukleären Mitosen und gelappten Zellkernen. Das primär zerebrale
Lymphom weist bei Patienten mit HIV-Infektion eine hohe Koinzidenz mit einer Epstein-Barr-Virus-Infektion auf, so dass dieser Nachweis im Liquor mittels PCR die Diagnose untermauern kann. Die Sensitivität für dieses diagnostische Verfahren beträgt $80 \%$, die Spezifität $90 \%$. Die endgültige Diagnosesicherung ist häufig jedoch erst durch stereotaktische Biopsie möglich.

Die Standardtherapie ist die Bestrahlung des Schädels mit einer Maximaldosis von $40 \mathrm{~Gy}$, die über drei Wochen fraktioniert wird. Medikamentös wird mit Kortison (z.B. mit Dexamethason) bei Meningeosis leucaemica additiv mit Methotrexat (15 mg 2 x pro Woche) intrathekal behandelt $(9,13)$. Nach Remission der Meningeosis lymphomatosa muss eine Erhaltungstherapie mit 15 mg Methotrexat monatlich intrathekal erfolgen. Ob eine operative Entfernung eines günstig gelegenen solitären primär zerebralen Lymphoms und eine Radiatio die Prognose verbessert, ist an großen Patientenkollektiven nicht untersucht. Unter HAART und Radiatio werden wesentlich längere Überlebenszeiten erreicht $(2,9,10,13)$, bei Bestrahlung mit bis zu 50 Gy des gesamten Neurokraniums und konsequenter HAART können Überlebenszeiten bis zu drei Jahren erreicht werden. Auch eine zytostatische Therapie mit Methotrexat (3 $\mathrm{g} / \mathrm{m}^{2} / 14 \mathrm{tgl}$.) intrathekal und systemisch oder eine Kombinationstherapie (Vincristin, Procarbazepin, Lomustin) kann zur Remission führen. Auch eine hochdosierte Kortisontherapie (Dexamethason 2x $40 \mathrm{mg} / \mathrm{d}$ ) wird gelegentlich angewandt.

Die konsequente Therapie erhöht die Überlebenszeit, ist gegenüber Patienten ohne HIV-Infektion jedoch deutlich verkürzt. Komplizierend treten oft nach erfolgreicher Behandlung des NHL opportunistische Infektionen auf, auch eine erhöhte Koinzidenz mit Schlaganfällen wird beobachtet.

\section{Immunrekonstitutions- erkrankungen}

Immunrekonstitutionserkrankungen stellen eine völlig neue Manifestationsform von neurologi- 
schen Erkrankungen kurz nach Beginn von HAART dar. Bei opportunistischen Infektionen wie z.B. der PML, der Kryptokokken-Meningitis oder aber auch bei Herpes-Infektionen tritt plötzlich eine Verschlechterung der Erkrankung bei ansteigenden CD4+-Zellen auf. Ursache ist eine irreguläre Immunrekonstitution mit Aspekten einer neu aufgetretenen Autoimmunerkrankung. Auch Leukoenzephalopathien, die ein Sarkoidose-ähnliches Bild aufweisen, werden beschrieben. Eine akute inflammatorische demyelinisierende Polyneuropathie ist ebenfalls möglich. Die Behandlung besteht in einer Attenuierung der Therapie mit HAART sowie gegebenenfalls in einer Kortison-Therapie.

\section{Opportunistic CNS Diseases in HIV and AIDS}

The introduction of highly active antiretroviral therapy (HAART) as standard treatment for HIV infection was followed by a drastic decrease in opportunistic infections. The incidence of newly acquired diseases of the central nervous system dropped from 5.9 per 100 persons in 1994 to 0.5 in 2002. Today, severe opportunistic infections in the highly developed industrialised countries are seen in patients with serious immune deficits or unrecognised HIV infections. Heading the list of opportunistic infections with focal lesions are toxoplasmosis and progressive multifocal leukoencephalopathy. Among the diffuse diseases, cerebral cryptococcosis is common, but in principle, the immunodeficiency syndrome facilitates the reactivation of any infections with which the patient has had contact. The differential diagnosis of opportunistic infections is based on neuroradiological investigations such as MRI and CSF examination using PCR. Nuclear medical methods are useful in the differentiation from primary cerebral lymphoma, the most important differential diagnosis of cerebral toxoplasmosis. In the case of opportunistic infections that cannot be adequately diagnosed with conventional conservative methods, the use of stereotactic brain biopsy makes sense and is indicated. Thanks to the combination of HAART and prompt rigorous treatment, the prognosis of opportunistic infections has been greatly improved.

\section{Key Words}

HAART - HIV-associated CNS diseases

- AIDS - encephalitis - lymphoma

\section{Literatur}

1. Akova-Öztürk E, Evers S, Colak-Ekici R, Heese C, Rickert CH, Reichelt D, Husstedt IW. Zur Korrelation klinisch-apparativer Differenzialdiagnosen mit neuropathischen Diagnosen bei Patienten mit AIDS. Nervenarzt 2004; 44: 930-932

2. Arendt G, von Giesen HJ. Klinik, Pathogenese und Therapie von Neuro-Aids. Bremen: Uni-Med-Verlag, 2000

3. Berger JR. Progressive Multifocal Leukencephalopathy. Current Treatment Opinion in Neurology 2000; 2: 361-368

4. Clifford BD. Focal brain lesions in people with HIV. Current Treatment Opinion in Neurology 1999; 1: 167-172

5. De Luca A, Giancola ML, Ammassari A et al. Cidofovir added to HAART improves virological and clinical outcome in AIDS-associated progressive multifocal leukencephalopathy. AIDS 2000; 29: F117-121

6. Dore GJ, McDonald A, Li Y, Kaldor JM, Brew BJ. Marked improvement in survival following AIDS dementia complex in the era of highly active antiretroviral therapy. AIDS 2003; 17: 1539-1545

7. Eggers Ch for the German Neuro-AIDS working group. HIV-1 associated encephalopathy and myelopathy. J Neurol 2002; 249: 1132-1136

8. Happe $\mathrm{S}$, Lüneborg $\mathrm{N}$, Rickert $\mathrm{CH}$, Heese C, Reichelt D, Schuierer G, Schul C, Husstedt IW. Progressive multifokale Leukenzephalopathie (PML) im Verlauf der AIDS-Erkrankung. - Übersicht und retrospektive Analyse von 17 Patienten. Nervenarzt 2000; 71: 96-104

9. Happe S, Milbradt O, Heese C, Oelerich M, Schul C, Reichelt D, Husstedt IW. Primäres Lymphom des zentralen Nervensystems als Neuromanifestation im AIDS-Stadium. Nervenarzt 2001; 72: 136-142

10. Husstedt IW. Primäre und sekundäre Neuromanifestationen der HIV-Infektion. Heidelberg: Springer Verlag, 2001

11. Lantos PL, McLaghlin JE, Scholtz CL, Berry CL, Tighe JR. Neuropathology of the brain in HIV-infection. The Lancet 1989; 1 8633: 309-311

12. D'Arminio Monforte $A$, Cinque $P$, Mocroft A, Goebel FD, Antunes F, Katlama C, Stenz Justesen U, Vella S, Kirk O, Lungren J. Changing Incidence of Central Nervous System Diseases in the EuroSIDA Cohort. Ann Neurol 2004; 55: 320-328

13. Deutsche Neuro-Aids-Arbeitsgemeinschaft (DNAA): Aktuelle Diagnostik und Therapie opportunistischer Hirnerkrankungen bei AIDS. DMW 2002; 127: 1479-1485 14. Ruiz A, Ganz WI, Donovan-Post JM: Use of thallium-201 brain-SPECT to differentiate cerebral lymphoma from toxoplasma encephalitis in AIDS patients. AJNR 1994; 15 : 1885-1894

15. Sacktor N, Lyles RH, Skolasky R, Kleeberger C, Selnes OA, Miller EN, Becker JT, Cohen B, McArthur JC. HIV-associated neurologic disease incidence changes: Multicenter $A$ Cohort Study, 1990-1998. Neurology 2001; 56(2): 257-260

16. Weltermann B, Röttgers HR, Lüdemann P, Evers S, Reichelt D, Husstedt IW. Kryptokokkenmeningoenzephalitis von HIV-infizierten Immigranten. Diagnostische unter therapeutische Aspekte unter Berücksichtigung der „evidence-based medicine“ - Ein Überblick. Nervenarzt 1999; 70: 364-400

Korrespondenzadresse: Prof. I.W. Husstedt Albert-Schweitzer-Str. 33 48129 Münster husstedt@uni-muenster.de 